\title{
Institut für Fort- und Weiterbildung in Klinischer
}

\section{Verhaltenstherapie e.V. (IFKV)}

Veranstaltungen im Sonderprogramm 1993

Sonderkurse

SK 5 Methodik und exemplarische Anwendung des EMG-Feedbacks

12.11.93:10-13 h, 14-17 h (8 UE) Prof. R. Höl·zl, Universität Mannheim

Ort: IFKV Bad Dürkheim

Gebühr: 160- DM (ermäßigt 120 - DM)

IFKV-Werkstatt

WS 2 Sexueller Mißbrauch: Therapeutische Erfahrungen mit Patientinnen in einem therapeutischen Setting

27.10.93:16-21 h (6 UE) Dipl.-Psych. B. Scheidt/Dipl.-Psych. D. Ecker Ort: IFKV Bad

Dürkheim Gebühr: frei

Ausbildung für Ausbilder

Af A 1 Das Kontaktgespräch in der Supervision

24.11.93: 16-21 h (6 UE) Dipl.-Psych. P. Kosarz/Dipl.-Psych. H. Lieb

Ort: IFKV Bad Dürkheim

Gebühr: 120- DM (ermäßigt 90 - DM)

Af A 2 Theorie und Praxis der Supervision

1.12.93:16-20 h (5 UE) Dr. W. A. Scobel

Ort: IFKV Bad Dürkheim

Gebühr: 100 - DM (ermäßigt 75 - DM)

Af A 3 Supervisionskonzeption im Kontext der Institution: Fallarbeit - Institutionsanalyse -

Selbstthematisierung

8.12.93:16-21 h (6 UE) Dr. K. Rappe-Giesecke Ort: IFKV Bad Dürkheim Gebühr: 120 - DM

(ermäßigt 90- DM)

Ermäßigung gilt für Mitglieder des IFKV sowie Absolventen und Teilnehmer der festen

Curricula im IFKV. Anmeldung unter Beifügung eines Schecks (für jeden Kurs gesondert).

Achtung:

Ab Dezember ist das Gesamtprogramm 1994 über das IFKV-Sekretariat erhältlich.

Ab 10/93 beginnt im IFKV ein neuer Ausbildungsgang zum «Verhaltenstherapeutischen

Kotherapeuten». Informationsmaterial können Sie über das Sekretariat beziehen.

Adresse:

IFKV-Sekretariat Kurbrunnenstraße 21a D-67098 Bad Dürkheim Tel. (06322)68019

Mitteilungen der Verbände 